\title{
Estrogens: possible protection against Amyotrophic Lateral Sclerosis?
}

Kátia de Vasconcelos ${ }^{1}$

Acary Souza Bulle Oliveira ${ }^{2}$

(iD) Luiz Fernando Portugal Fuchs ${ }^{1}$

(iD) Ricardo Santos Simões ${ }^{3}$

Manoel loão Batista Castello Girão

(iD) Jose Maria Soares Júnior ${ }^{3}$

(iD) Edmund Chada Baracat ${ }^{3}$

\begin{abstract}
1. Department of Gynecology, Escola Paulista de Medicina/Universidade Federal de São Paulo; São Paulo, Brasil 2. Department of Neurology and Neurosurgery, Escola Paulista de Medicina/Universidade Federal de São Paulo; São Paulo, Brasil 3. Department of Gynecology and Obstetrics, Hospital das Clínicas, School of Medicine of the University of São Paulo; São Paulo, Brasil
\end{abstract}

http://dx.doi.org/10.1590/1806-9282.65.5.576

Amyotrophic Lateral Sclerosis (ALS) is a neurodegenerative disease characterized by progressive degeneration of upper and lower motor neurons in the motor cortex and spinal cord ${ }^{1,2}$. It can be sporadic, which corresponds to $90 \%$ of cases, or familial, mostly autosomal dominant, corresponding to $10 \%$ of cases. The disease's initial symptoms are local muscle weakness, progressing to rigidity and paralysis, also involving swallowing, diaphragm, which lead patients to death in 2 to 5 years due to respiratory failure ${ }^{1,2}$.

In both etiology and physiopathogeny, there are reports of the influence of sex steroids. Epidemiologically, the disease's onset is later in women, around their 70 years, while in men approximately with 55 years ${ }^{1-4}$.

In fact, gender and age exert a great influence on the risk of developing ALS, where the proportion be- tween men and women would be between 4:1 in the period between 30 and 50 years of age. With the aging process and hypoestrogenism, this difference between the sexes decays to a ratio of 1:1 after age 65, leading to the idea that sex hormones can participate in onset, evolution, as well as intensity ${ }^{1}$.

Due to aging-related changes, both estrogen synthesis and the expression of its receptors may differ based on cell and tissue types, an important aspect to understand the roles of cellular and tissue-specific hormonal synthesis to diseases that have being related to age ${ }^{5,6}$.

The brain is a tissue responsive to the sexual hormone, and therefore affected by the drastic fall of estrogenic synthesis in menopause, a fact already associated with numerous affections, among these cognitive diseases such as Alzheimer's and other dementias, as well as recent studies pointing out to a possible 
relation of the sexual hormones with the ALS, where estrogens would possibly act as a neuroprotector ${ }^{5,6}$.

There is evidence suggesting that the concentration of some specific hormones, such as estrogens and progesterone, or the expression of their receptors, may play an important role on the prevalence, progression, and survival of those patients ${ }^{1,5}$.

This reality changes in terms of hormone replacement therapy, mainly estrogens, which do not have positive effects in postmenopausal women as in ALS. Also, the survival period will not change. It may be questioned that after neuronal damage, there is no therapy that can reverse the process ${ }^{1}$.

There are other differences between the genres, among which, the clinical form: in men, the most frequent is in the upper limbs, whereas in women, the bulbar form is the predominant one. Another important factor is the possible increase in the risk of ALS for women who presented late menarche and early menopause $^{3}$.

There are some possible protective actions for estradiol (E2), such as the direct promotion of cell survival, preventing cell death by acting directly on the cascade of the apoptosis process. Estradiol is responsible for controlling the differentiation and plasticity of different neuronal populations in brain development, such as potentiating neurogenesis, modulating synaptogenesis and, finally, influencing axonal budding ${ }^{5,6}$. E2 also regulates neurotransmitter activ-

\section{REFERENCES}

1. Blasco $H$, Guennoc $A M$, Veyrat-Durebex $C$, Gordon PH, Andres CR, Camu W, et al. Amyotrophic lateral sclerosis: a hormonal condition? Amyotroph Lateral Scler 2012; 13(6):585-8. doi: 10.3109/17482968.2012.706303.

2. Kiernan MC, Vucic S, Cheah BC, Turner MR, Eisen A, Hardiman O, et al. Amyotrophic lateral sclerosis. Lancet 2011; 377(9769):942-55. doi: 10.1016/ S0140-6736(10)61156-7.

3. Aggarwal T, Polanco MJ, Scaramuzzino C, Rocchi A, Milioto C, Emionite $L$ et al. Androgens affect muscle, motor neuron, and survival in a mouse model of SOD1-related amyotrophic lateral sclerosis. Eurobiol Aging 2014; 35(8):1929-38. doi: 10.1016/..neurobiolaging.2014.02.004. ity and their receptors among different populations of neurons. Therefore, it would have positive effects on neurological preservation, as long as the damage was not intense ${ }^{5.6}$.

Other estrone protective actions would be the antioxidant action, removing reactive oxygen species, and anti-inflammatory, where it would act to diminish the production of TNF- $\alpha$, IL- $1 \beta$ and IL- $6{ }^{1}$. On the other hand, inflammatory processes in the central nervous system would stimulate the production of inflammatory mediators in response to estrogen: cytokines (produced mainly by glial cells), stimulate the immune system and can induce the death of neurons. This fact could explain why the hormone therapy did not improve ALS after its onset ${ }^{1,6}$.

The mechanisms by which estrogen would exercise its protective action and benefit on the neurological system have not been fully known, but their action may be preventive, i.e, it would have no effect when the neurological disease, such as ALS, is already installed, and may even worsen it. In addition, progesterone in hormone therapy has negative action of motor plate, which would also be a negative influence on $\mathrm{ALS}^{6}$.

Finally, postmenopausal hormone replacement therapy could improve connection and neurological protection when prescribed early, but belatedly, it would not have benefits over established degenerative diseases such as ALS ${ }^{\mathbf{1 5 , 6}}$.

4. Manjaly ZR, Scott KM, Abhinav K, Wijesekera L, Ganesalingam J, Goldstein $\mathrm{LH}$, et al. The sex ratio in amyotrophic lateral sclerosis: A population based study. Amyotroph Lateral Scler 2010;11(5):439-42. doi: 10.3109/17482961003610853.

5. de Jong S, Huisman M, Sutedja N, van der Kooi A, de Visser M, Schelhaas |, et al. Endogenous female reproductive hormones and the risk of amyotrophic lateral sclerosis. J Neurol 2013; 260(2):507-12. doi: 10.1007/ s00415-012-6665-5.

6. Garcia-Segura LM, Azcoitia I, DonCarlos LL. Neuroprotection by estradiol. Prog Neurobiol 2001; 63(1):29-60. https://doi.org/10.1016/S03010082(00)00025-3. 